Article

\title{
Centrifugation Conditions in the L-PRP Preparation Affect Soluble Factors Release and Mesenchymal Stem Cell Proliferation in Fibrin Nanofibers
}

\author{
Bruna Alice Gomes de Melo ${ }^{1}$, Ângela Cristina Malheiros Luzo ${ }^{2}$, José Fabio Santos Duarte Lana ${ }^{3}$ \\ and Maria Helena Andrade Santana 1,*(D) \\ 1 Department of Engineering of Materials and Bioprocesses, School of Chemical Engineering, \\ University of Campinas, Campinas, SP 13083-852, Brazil \\ 2 Hematology \& Hemotherapy Center, Umbilical Cord Blood Bank, University of Campinas, \\ Campinas, SP 13083-878, Brazil \\ 3 Bone and Cartilage Institute, Indaiatuba, SP 13334-170, Brazil \\ * Correspondence: lena@feq.unicamp.br; Tel.: +55-19-35213921
}

Academic Editors: Marco Rossi, Daniele Passeri and Francesca A. Scaramuzzo

check for Received: 10 June 2019; Accepted: 19 July 2019; Published: 27 July 2019

updates

\begin{abstract}
Leukocyte and platelet-rich plasma (L-PRP) is an autologous product that when activated forms fibrin nanofibers, which are useful in regenerative medicine. As an important part of the preparation of L-PRP, the centrifugation parameters may affect the release of soluble factors that modulate the behavior of the cells in the nanofibers. In this study, we evaluated the influences of four different centrifugation conditions on the concentration of platelets and leukocytes in L-PRP and on the anabolic/catabolic balance of the nanofiber microenvironment. Human adipose-derived mesenchymal stem cells (h-AdMSCs) were seeded in the nanofibers, and their viability and growth were evaluated. L-PRPs prepared at $100 \times g$ and $100+400 \times g$ released higher levels of transforming growth factor (TGF)- $\beta 1$ and platelet-derived growth factor (PDGF)-BB due to the increased platelet concentration, while inflammatory cytokines interleukin (IL)-8 and tumor necrosis factor (TNF)- $\alpha$ were more significantly released from L-PRPs prepared via two centrifugation steps $(100+400 \times g$ and $800+400 \times g$ ) due to the increased concentration of leukocytes. Our results showed that with the exception of nanofibers formed from L-PRP prepared at $800+400 \times g$, all other microenvironments were favorable for h-AdMSC proliferation. Here, we present a reproducible protocol for the standardization of L-PRP and fibrin nanofibers useful in clinical practices with known platelet/leukocyte ratios and in vitro evaluations that may predict in vivo results.
\end{abstract}

Keywords: platelet; leukocyte; L-PRP; centrifugation; fibrin; nanofiber; growth factor; cytokine; mesenchymal stem cells

\section{Introduction}

In the past few years, the benefits of autologous leukocyte- and platelet-rich plasma (L-PRP) have been evidenced in the treatment of many types of diseases [1-6]. Aside from growth factors (GFs) released from the platelets' alpha granules, L-PRP contains inflammatory cytokines secreted from leukocytes that act in synergy to modulate the migration, proliferation, and differentiation of autologous cells through different pathways that lead to tissue regeneration [7-11]. Depending on the site, the degree of the injury (acute or chronic), and treatment phase (early or late stage of healing), the leukocyte fraction must be adjusted from poor-leukocyte PRP (P-PRP) to L-PRP [12-14]. Modern classifications systems consider the platelet and leukocyte levels, aside from other conditions, such as the number of centrifugation spins, activation, the presence of erythrocytes, and guided applications [15-18]. 
Whether conducted manually or by machine, L-PRP is prepared by centrifuging the patient's whole blood, in which platelets, leukocytes, proteins, and other components are concentrated in a small fraction of plasma, with their levels adjusted by varying the centrifugation conditions [19,20]. In a previous study, we determined the distribution and recovery of platelets and leukocytes (lymphocytes and granulocytes) in the supernatant, buffy coat, and erythrocyte layers by centrifuging the whole blood from 100 to $800 \times g\left(10 \mathrm{~min}\right.$ and $\left.25^{\circ} \mathrm{C}\right)$. The concentration patterns allowed for the identification of specific centrifugation ranges to obtain distinct platelet/leukocyte and the associate lymphocyte/granulocyte ratios. As blood centrifugation is a separation process based on the size and density of the components as well as the packing behavior of the erythrocytes, different recoveries of platelets and leukocytes could be obtained in L-PRP from a first spin [19]. In turn, a second centrifugation step under accelerations higher than $400 \times g$ could lead to platelet aggregation and deactivation [20,21]. Therefore, choosing the centrifugation conditions for L-PRP preparation is crucial to achieving an optimized performance.

When activated, L-PRP forms a 3D network composed of fibrin nanofibers that act as a scaffold for autologous cells to migrate, proliferate, and differentiate at the injury site. Various works in the literature have used fibrin nanofibers as a scaffold for tissue engineering, whether obtained from commercial fibrinogen [22-24] or from PRP formulations [25-27], and have reported on their suitability in regenerative medicine. However, fibrin from PRP presents advantages over commercial fibrin due to its rich microenvironment, with many GFs and cytokines being gradually released from the nanofibers, thus stimulating the regenerative process $[28,29]$. The PRP type and preparation conditions directly influence the final architecture of the fibrin networks in terms of the nanofiber thickness, length, and organization [30]. While P-PRP and L-PRP favor the formation of low-density fibrin, P-PRF and L-PRF (pure platelet-rich fibrin and leukocyte- and platelet-rich fibrin) favor the formation of high-density fibrin [16], which could affect the GFs and the entrapment and release dynamics of the cytokines [28].

Transforming GF (TGF- $\beta$ ) and platelet-derived GF (PDGF) are the most abundant GFs present in the platelets' alpha granules and are primarily responsible for modulating the wound healing process [31]. They act in the early stages of healing by creating a concentration gradient to attract inflammatory agents, macrophages, mesenchymal stem cells (MSCs), fibroblasts, and other cells to the injury site, and also stimulate the secretion of more GFs, cell proliferation, differentiation, and the production of extracellular matrix (ECM) [11,32,33].

Leukocytes are known to secrete inflammatory cytokines, such as interleukin (IL)-8 and tumor necrosis factor (TNF)- $\alpha$, which leads to ECM degradation [34,35]. However, they play essential roles in healing during the inflammatory phase, acting as immunoregulatory and antibacterial agents, as well balancing the anabolic/catabolic microenvironment [10,36-38]. Therefore, an optimized concentration of cytokines is crucial for contributing to the regenerative process and the maintenance of tissue homeostasis [37,39,40].

Associated with endogenous MSCs, L-PRP and fibrin nanofibers constitute the proliferation triangle, a term previously created to identify all necessary components to achieve effective tissue regeneration (3D matrix, signaling molecules, and progenitor cells) [31,41]. Due to its high availability and great chondrogenic and osteogenic capability, exogenous MSCs have been used in cell-therapies and cartilage and bone engineering studies, presenting synergistic effects with L-PRP, such as increased GF and cytokine secretion, cell migration, proliferation, and differentiation [42-44].

In this work, we aimed to study the effects of different centrifugation conditions on the preparation of L-PRP, the release kinetics of GFs and cytokines, and on the response of MSCs from human adipose-derived tissue (h-AdMSCs) seeded in fibrin nanofibers. The results obtained represent an advanced step toward understanding the effects of the platelet/leukocyte ratio and catabolic/anabolic balance on cell behavior. The standardized preparations reduce variability and advise physicians and researchers to select the parameters and operational conditions to obtain L-PRP and fibrin nanofibers with optimal yield and performance. 


\section{Results}

L-PRP was prepared by centrifuging the whole blood at two accelerations, 100 and $800 \times \mathrm{g}$, using one or two spin steps to form four formulations: L-PRP $100, \mathrm{~L}_{-\mathrm{PRP}} \mathrm{P}_{100-400}, \mathrm{~L}_{-\mathrm{PRP}} \mathrm{P}_{80}$, and L-PRP $800-400$ (Figure 1A). L-PRP activation led to the formation of fibrin nanofibers (fibrin 100, fibrin $_{100-400}$, fibrin $_{800}$, and fibrin $800-400$ ) rich in GFs and cytokines that are suitable for supporting cells, and whose response depend on the anabolic/catabolic balance of the microenvironment (Figure 1B).

A

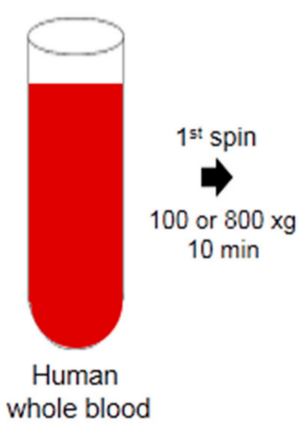

B

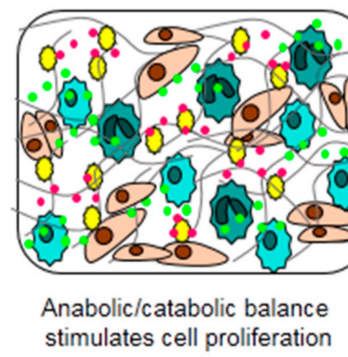

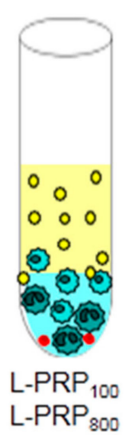
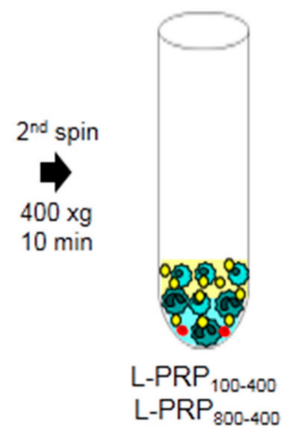
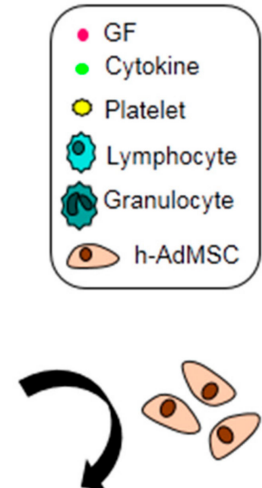

Activation with thrombin $/ \mathrm{Ca}^{2+}$

GFs and cytokine gradual release

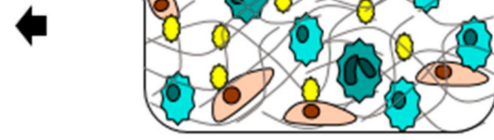

3D network of fibrin nanofibers

(fibrin ${ }_{100}$, fibrin $100-400$, fibrin $_{300}$ and fibrin $n_{800-400}$ )

Figure 1. Schematic illustration of the experimental design. (A) Human whole blood was collected and centrifuged at 100 and $800 \times \mathrm{g}$ for $10 \mathrm{~min}$ to form L-PRP 100 and L-PRP 800 . These L-PRPs were submitted to a second spin at $400 \mathrm{xg}$ for $10 \mathrm{~min}$ to form L-PRP $100-400$ and L-PRP $800-400$ after the removal of $70 \%$ of the supernatant; (B) h-AdMSCs were resuspended in each L-PRP, followed by the formation of a fibrin nanofiber network after activation with thrombin/ $\mathrm{Ca}^{2+}$. Activated platelets and leukocytes secrete GFs and cytokines that in an appropriate balance stimulate cell proliferation.

The concentrations of platelets, total leukocytes, lymphocytes, and granulocytes in the whole blood were $149 \pm 33,3.8 \pm 0.9,1.4 \pm 0.9$, and $2.3 \pm 0.4$, respectively. The slower acceleration $(100 \times g)$ allowed the recovery of an increased concentration of platelets, especially after two centrifugation steps (Figure 2A), as also observed for the total leukocytes (Figure 2B). Lymphocytes represented the highest leukocyte portion, being highly concentrated in L-PRP $100-400$ (Figure 2C). On the other hand, the granulocyte concentration was higher in L-PRP 800 and L-PRP $800-400$ when compared to L-PRPs from $100 \times g$ (Figure 2D). Therefore, the platelet/leukocyte and lymphocyte/granulocyte ratios were greater in both L-PRP 100 and L-PRP $100-400$ in comparison to L-PRP 800 and L-PRP $800-400$ (Figure 2E,F).

The concentration factor of all components in each L-PRP was calculated relative to the whole blood, and values varied according to the applied centrifugation condition (Figure $3 \mathrm{~A}$ ). The highest value was obtained for platelets in L-PRP 100 and L-PRP $100-400$, which were $1.98 \pm 0.2$ and $2.8 \pm 0.3$, respectively. The high speed significantly decreased the concentration factor of the platelets, which did not reach the baseline at $\sim 0.15$ for L-PRP 800 and $\mathrm{L}-\mathrm{PRP}_{800-400}$. At this speed, the concentration factor was below 1 for these two formulations due to the low concentration of granulocytes. However, a significant increase in this component was observed after the second centrifugation, which was $0.17 \pm 0.02$ and $0.22 \pm 0.03$ for L-PRP $100-400$ and L-PRP $800-400$, respectively. Among all formulations, the highest concentration factor of lymphocytes was observed in L-PRP ${ }_{100-400}(1.35 \pm 0.2)$ (Figure 3B). 

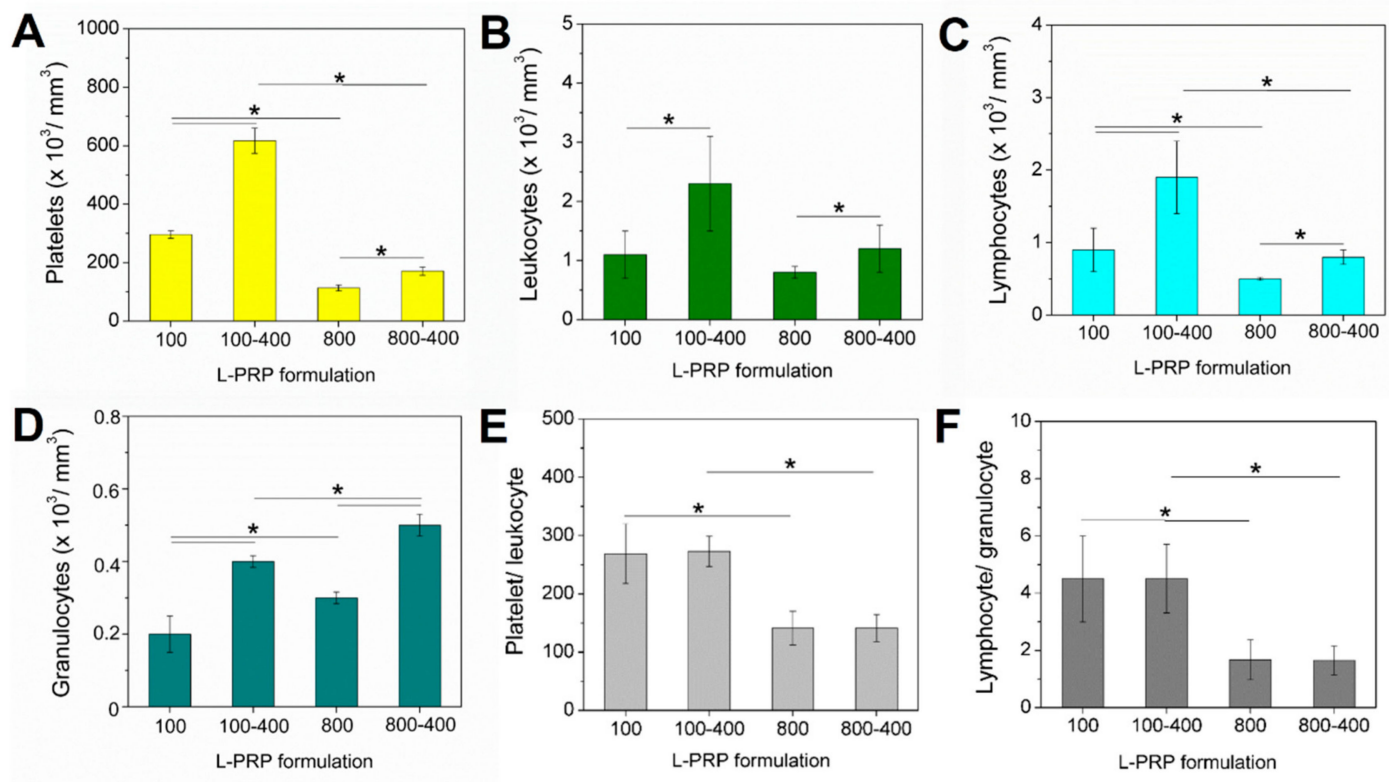

Figure 2. Concentration of (A) platelets, (B) leukocytes, (C) lymphocytes, and (D) granulocytes, obtained by measuring each L-PRP in a hematologic analyzer. The calculated ratio of (E) platelet/leukocyte and (F) lymphocyte/granulocyte. Note: ${ }^{*} p<0.05$.
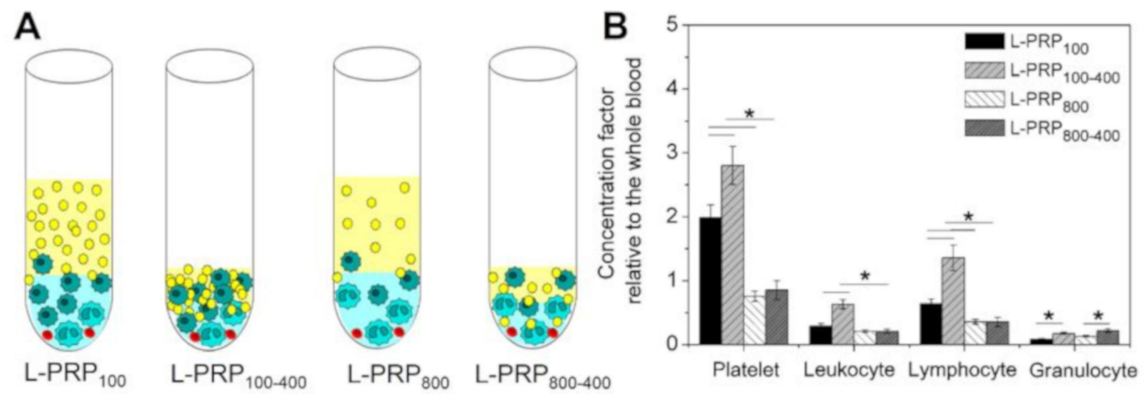

Figure 3. (A) Schematic illustration representing the concentration of the blood components in each L-PRP. (B) The calculated concentration factors relative to the whole blood. Note: ${ }^{*} p<0.05$.

Fibrin nanofibers were formed after the activation of L-PRPs with thrombin/ $\mathrm{Ca}^{2+}$. The scanning electron microscopy (SEM) images showed no substantial changes in morphology, with the structures presenting high porosity and interconnected nanofiber organization (Figure 4A). For all samples, the fiber diameters varied from 100 to $200 \mathrm{~nm}$, with less polydispersity being observed for nanofibers prepared with L-PRPs via two centrifugation steps (Figure 4B).

The cumulative concentration of GFs and cytokines released from each network was measured over $72 \mathrm{~h}$ (Figure 5). It was observed that GFs released from nanofibers increased proportionally with an increase in platelets in L-PRP. PDGF-BB presented a fast release in the first $3 \mathrm{~h}$ for fibrin 100 and fibrin $_{100-400}$, reaching $4230 \pm 234$ and $6290 \pm 150 \mathrm{pg} / \mathrm{mL}$ at $72 \mathrm{~h}$, respectively. For fibrin $800-400$, the release was slower, but concentration reached a high level at $72 \mathrm{~h}$ at $3335 \pm 65 \mathrm{pg} / \mathrm{mL}$. TGF- $\beta 1$ presented the highest level among all GFs, as it was gradually released over time. Its maximum concentration was $8576 \pm 305,11,315 \pm 840,1092 \pm 47$, and $4490 \pm 545 \mathrm{pg} / \mathrm{mL}$ for fibrin ${ }_{100}$, fibrin $_{100-400}$, fibrin $_{800}$, and fibrin $800-400$, respectively (Figure 5A). The PDGF-BB concentration released from fibrin 800 was the smallest and reached equilibrium rapidly, with values remaining below $1000 \mathrm{pg} / \mathrm{mL}$ (Figure 5B). 
A
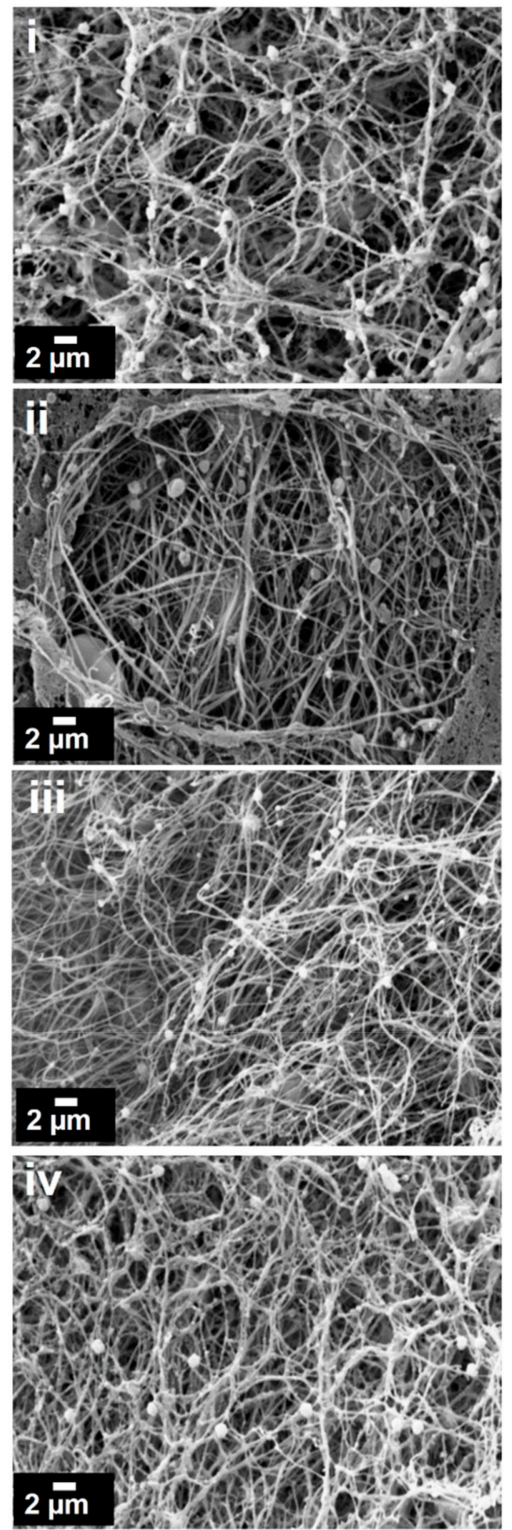
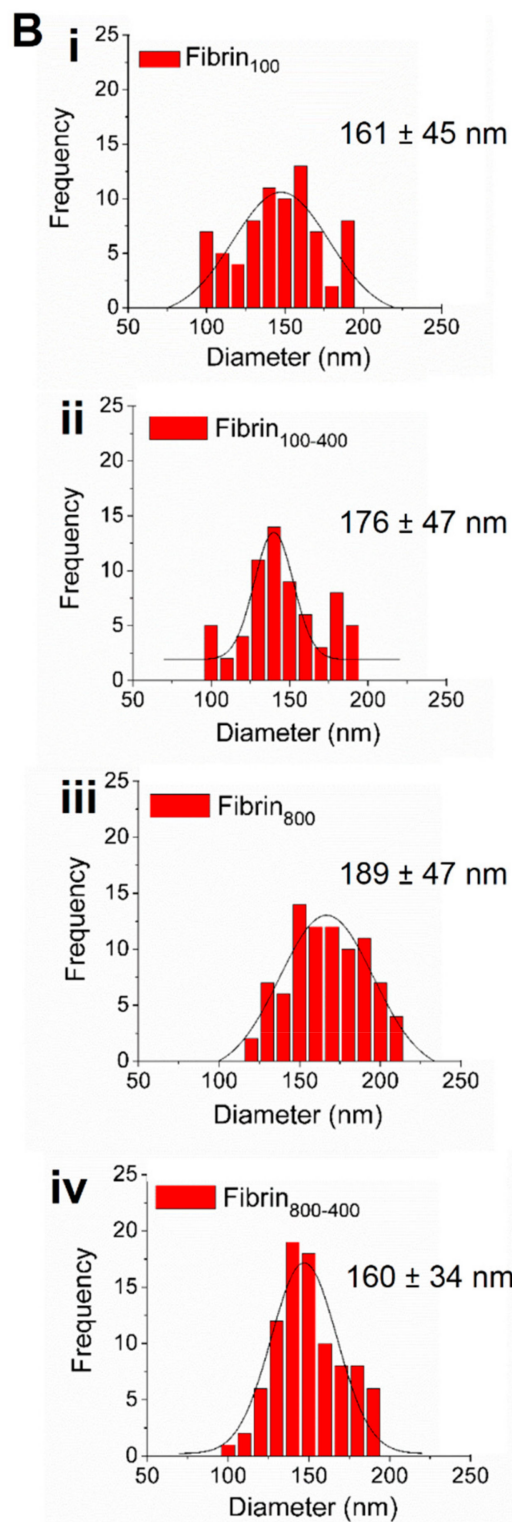

Figure 4. Characterization of the fibrin nanofibers. (A) SEM images showing the morphology of the networks. (B) Frequency of fiber diameter measured using ImageJ software, highlighting their mean diameter; (i) fibrin 100 , (ii) fibrin $100-400$, (iii) fibrin 800 , and (iv) fibrin $800-400$.

The inflammatory cytokine IL-8 was highly secreted, and its concentration increased with the increase in leukocytes in L-PRP (Figure 5C). In the first $24 \mathrm{~h}$, fibrin $100-400$ and fibrin $800-400$ presented a similar trend of release kinetics. Then, the IL- 8 secretion sharply increased for fibrin $100-400$, reaching $3490 \pm 70 \mathrm{pg} / \mathrm{mL}$ at $72 \mathrm{~h}$, while for fibrin ${ }_{800}-400$, the value was $2382 \pm 136 \mathrm{pg} / \mathrm{mL}$. For fibrin 100 , the IL-8 kinetics was slow up to $24 \mathrm{~h}$, then increased sharply after this time, reaching $1013 \pm 420 \mathrm{pg} / \mathrm{mL}$. The concentration of this cytokine in fibrin 800 was below $200 \mathrm{pg} / \mathrm{mL}$. TNF- $\alpha$ was significantly higher when released from fibrin $100-400$ in comparison to the other formulations during all of the times studied, and was $\sim 2.5$-fold and $\sim 4$-fold greater than fibrin $800-400$ and fibrin ${ }_{100}$, respectively (Figure 5D). Similar to IL-8, the concentration of TNF- $\alpha$ released from fibrin $_{800}$ presented the smallest value $(<100 \mathrm{pg} / \mathrm{mL})$.

The L-PRP formulations were also prepared using the whole blood of two other different donors (donor 2 and donor 3), and even though they showed an initial concentration of platelets and leukocytes that were significantly different from the current donor (Figure 2 and Figure S1, Supplementary Materials), the results presented a similar trend for GF and cytokine release (Figure 5 and Figure S2, 
Supplementary Materials). GF concentration was proportional to the platelet/leukocyte ratio, whereas cytokines were proportional to the lymphocyte/granulocyte ratio. The lowest lymphocyte/granulocyte ratio observed for the current donor was reflected in the highest levels of TNF- $\alpha$ due to the predominance of granulocytes, as compared to donors 2 and 3.
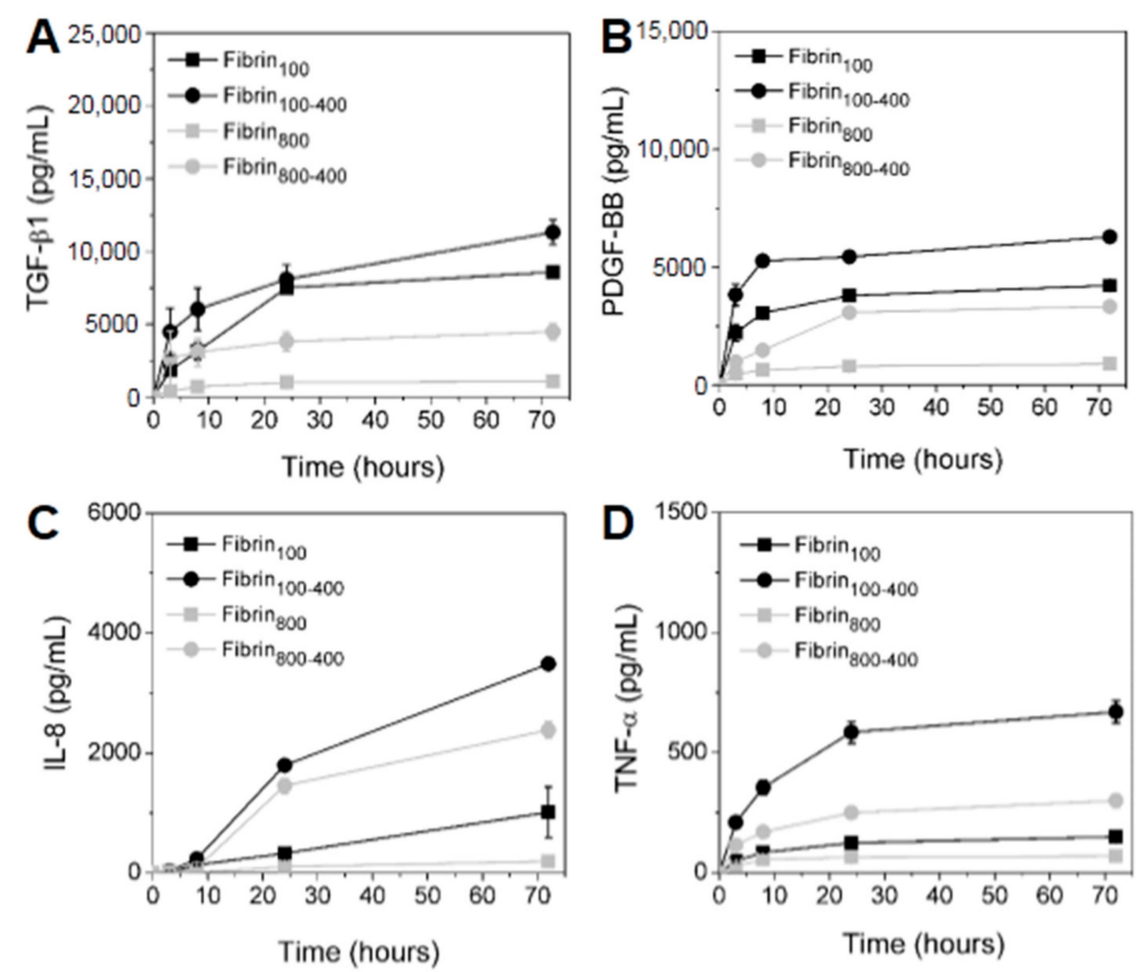

Figure 5. Kinetics of the GFs (A) TGF- $\beta 1$, (B) PDGF-BB, and the inflammatory cytokines (C) IL-8 and (D) TNF- $\alpha$ released from the nanofibers over $72 \mathrm{~h}$.

The h-AdMSCs were cultured in the nanofibers, and their behavior in the different microenvironments was assessed as the viability and proliferation rate. Images of living and dead cells were captured in a fluorescence confocal microscope using live/dead reagents after seven days of culture. Results showed that the fibrin networks were suitable to maintain cell viability, with h-AdMSCs presenting a slightly elongated to rounded shape within the networks (Figure 6A). On day 7, the number of viable cells in the samples prepared with gentle centrifugal acceleration was significantly higher than that in the samples prepared at higher speed, with viability being $87.2 \pm 2.9,86.1 \pm 2.8$, $75.6 \pm 0.6$, and $74.3 \pm 3 \%$ for fibrin 100 , fibrin $100-400$, fibrin 800 , and fibrin $800-400$, respectively (Figure 6B).

Figure 6C shows the proliferation kinetics of h-AdMSCs in terms of absorbance (595 nm) with time, which allowed for the estimation of the cell adaptation time (before the exponential phase), the range of the exponential phase, $\mu_{\max }$, and $t_{d}$ for the $h$-AdMSCs seeded in each preparation (Table 1). Values of estimated $t_{d}$ were in accordance with the doubling of absorbances, approximately, as shown in Figure 6C. The highest adaption time was observed for fibrin $800-400$, while the lowest was for fibrin $_{100-400}$. The exponential phase was approximately 4-5 days for all conditions. Although $\mu_{\max }$ and $t_{d}$ could not be estimated because it tended to be out of the studied range $(<3)$, the values for the other conditions were similar, except for fibrin 800 , where the $t_{d}$ was slightly higher. 
Table 1. Kinetic parameters for h-AdMSC proliferation seeded in the fibrin networks of the L-PRP preparations.

\begin{tabular}{lcccc}
\hline \multicolumn{1}{c}{ Condition } & $\begin{array}{c}\text { Adaptation Time } \\
\text { (Day) }\end{array}$ & $\begin{array}{c}\text { Range of Exponential } \\
\text { Phase (Day) }\end{array}$ & $\boldsymbol{\mu}_{\max } *\left(\right.$ day $\left.^{-\mathbf{1}}\right)$ & $\mathbf{t}_{\mathbf{d}}{ }^{* *}$ (day) \\
\hline Fibrin $_{\mathbf{1 0 0}}$ & $\sim 4$ & $4-9$ & 0.15 & 4.53 \\
\hline Fibrin $_{\mathbf{1 0 0}-400}$ & $<<3$ & $<3$ & $* * *$ & $* * * *$ \\
\hline Fibrin $_{\mathbf{8 0 0}}$ & $\sim 3$ & $3-7$ & 0.16 & 4.33 \\
\hline Fibrin $_{\mathbf{8 0 0}-400}$ & $\sim 6$ & $6-10$ & 0.14 & 4.86 \\
\hline
\end{tabular}

Note: ${ }^{*} \mu_{\max }=$ maximum proliferation rate determined in the exponential phase; ${ }^{* *} t_{d}=$ doubling time calculated from the beginning of the exponential phase; ${ }^{* * *}$ not estimated. Out of the range of the experimental data.
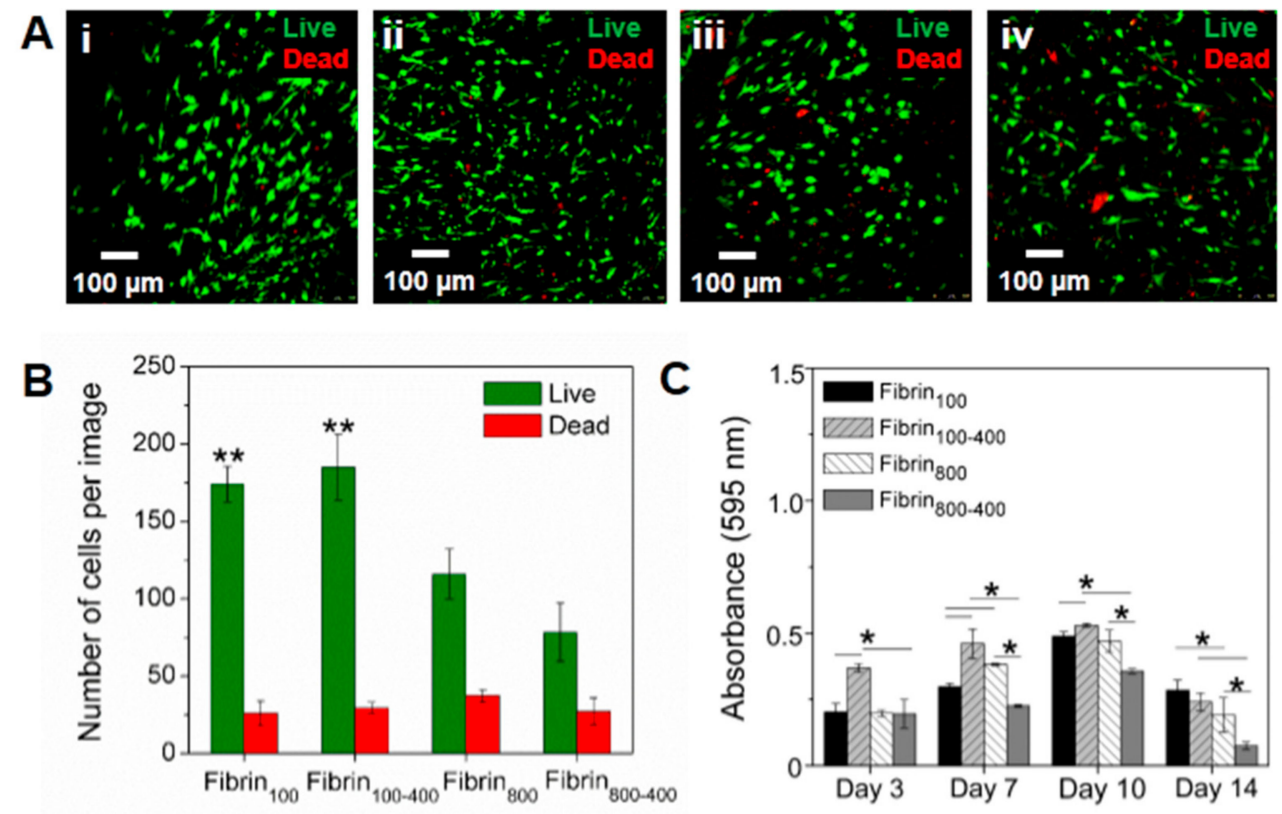

Figure 6. Assessment of h-AdMSC behavior seeded in the four types of fibrin nanofibers. (A) Cell viability on the seventh day of incubation in (i) fibrin $_{100}$, (ii) fibrin $100-400$, (iii) fibrin 800 , and (iv) fibrin $_{800-400}$. (B) Number of living and dead h-AdMSCs in the nanofibers incubated for seven days and calculated from three different images using ImageJ software. Note: ${ }^{* *}$ Significantly different from fibrin $_{800}$ and fibrin $_{800-400}(p<0.05)$. (C) Proliferation kinetics for h-AdMSCs seeded in the fibrin networks of the L-PRP preparations. Note: ${ }^{*} p<0.05$.

\section{Discussion}

In previous work, we reported on the mechanisms of platelet and leukocyte recovery in L-PRP by centrifugation, which is driven by size, density, and erythrocyte aggregation and sedimentation [19]. As predicted by theoretical calculations and experimentally confirmed, low centrifugal accelerations in the first spin (50-100 $\times$ g) contribute to high yields of platelets in P-PRP (upper layer) [45]. If high spin ranges were used up until the 2010s [46,47], nowadays, we can see that low speeds for a first spin have become the consensus [21,48,49]. At the lower range of centrifugal accelerations, we have also observed the highest leukocyte recovery, with increased platelet/leukocyte and lymphocyte/granulocyte ratios in L-PRP, in comparison to accelerations ranging from 300 to $800 \times g$ [19]. As microenvironments with distinct blood component ratios lead to different biological responses, the standardization of centrifugation conditions and the study of cell responses to different types of L-PRPs is essential in predicting its behavior in vivo.

L-PRP activation leads to fibrin clot formation, a network composed of fibrin nanofibers that controls the release of soluble factors into the microenvironment and supports cell attachment, proliferation, differentiation, and the formation of new tissue [50]. As different centrifugal accelerations 
and leukocyte concentrations are related to morphological changes in the nanofibers [25,28,49], we analyzed the network's morphology by SEM. Here, we observed that for all conditions, interconnected nanofibers were formed with a random organization, along with networks with high porosity. The different concentrations of blood components did not affect the fibrin morphology, therefore, we assumed that this would not interfere in the kinetic release of GFs and cytokines.

Gentle centrifugal acceleration is essential to maintain platelet integrity and retain their morphological properties and activation capacity, which is crucial for their gradual content release mechanism. Under high centrifugal forces, platelets are submitted to high shear stress that can induce aggregation, activation, and early GF release, impairing the proper functioning of L-PRP [20,21,49]. As previously reported, platelet activation increases with an increase in centrifugation speed, resulting in changes in their biological signature [21]. Here, we observed that the release of PDGF-BB and TGF- $\beta 1$ was proportional to platelet concentration, which was higher for fibrin ${ }_{100}$ and fibrin $100-400$. For fibrin $_{800}$, the concentration of both GFs was minimum. However, the first spin at $800 \times g$ did not damage the platelets, and after the second centrifugation at $400 \times g$, an increased and gradual release of PDGF-BB and TGF- $\beta 1$ was observed, indicating the maintenance of the platelets' integrity. These GFs were selected for the experiment as they were the most abundant in the platelets, and were crucial in the anabolic pathways of the regeneration process by stimulating cell proliferation, differentiation, and the formation of extracellular matrix (ECM) [37,51].

Leukocytes secrete cytokines upon activation and strongly depend on the stimulus from the microenvironment [34,52]. Here, we observed that IL-8 and TNF- $\alpha$ were highly released from fibrin $_{100-400}$ and fibrin $_{800-400}$, with the concentration proportional to the number of leukocytes. As previously reported, granulocytes are capable of synthesizing and secreting TNF- $\alpha$ independent of stimulus [53], thus corroborating our results. TNF- $\alpha$ is immunostimulatory to lymphocytes and modulates the secretion of other cytokines, such as IL-8 [34]. It is also a potent neutrophil chemoattractant, recruiting these granulocytes to inflammatory sites for debridement and to fight infections [54].

By performing the experiment using the L-PRPs of different donors, we showed that the kinetic release of GFs and cytokines followed a trend based on the centrifugation condition applied, regardless of the initial platelet and leukocyte concentration. Therefore, it can be considered that our protocol is reproducible and suitable for application in clinical practice.

Although the presence of leukocytes, especially that of granulocytic neutrophils, could be harmful to cells due to their catabolic effects [38], by assessing the response of h-AdMSCs to the different microenvironments, we attested the role of anabolic/catabolic balance. Cell growth was modulated by the stimulus provided by the GFs, especially PDGF-BB and TGF- $\beta 1$, which are important in the signaling pathways of MSC proliferation [55] and the inhibition of cytokines, with the anabolic/catabolic (GFs/cytokines) balance playing a major role in cell behavior [39]. The greatest proliferation stimulus was provided by the nanofibers fibrin 100 and fibrin $100-400$, while inhibition was stronger for fibrin $800-400$. These results are in accordance with the kinetic release of GFs and cytokines, where fibrin $100-400$ showed a higher GF concentration and a trend for the lowest adaption time, while the high concentration of inflammatory cytokines and the lowest concentration of GFs in fibrin $800-400$ resulted in an imbalance of the anabolic/catabolic ratio, with cells presenting the highest adaptation time. As fibrin 800 released the lowest concentration of inflammatory cytokines, even presenting lower levels of released TGF- $\beta 1$ and PDGF-BB, its maximum absorbance on day 10 was slightly less than those of fibrin 100 and fibrin $_{100-400}$. In all fibrin networks, h-AdMSCs were in the death phase on day 14, probably due to the lack of physical space for cell duplication or nutrient depletion.

We concluded that rather than the absolute values of platelets or leukocytes, the platelet/leukocyte ratio drove cell behavior through the balance between the anabolic/catabolic effects. This balance is altered during injury and is reconstituted by L-PRP components, where the microenvironment returns to a beneficial balance and homeostasis. Therefore, it could be inferred that anabolic/catabolic balance 
might play a major role in the mechanisms that lead to h-AdMSC proliferation, which has a direct impact on tissue regeneration.

These results are coherent with the consensus that considers that the higher the concentration of platelets, the greater the anabolic effects [15]. However, we recommend a balanced platelet/leukocyte ( $>200)$ and the correspondent lymphocyte/granulocyte ratio $(>1)$, which could present a higher efficiency in the applications of L-PRP and its nanofiber network in regenerative medicine.

\section{Material and Methods}

\subsection{Whole Blood Collection}

The Ethics Committee of the Medical Sciences School of the University of Campinas, Campinas, SP, Brazil (UNICAMP) (CAAE: 0972.0.146.000-11) approved the use of human blood in this study, which was collected from a healthy donor in a tube containing anticoagulant acid citrate dextrose solution A (ACD-A) (BD Vacutainer ${ }^{\circledR}$, Dade City, FL, USA) prior to centrifugation.

\subsection{L-PRP Preparation}

Centrifugation of human whole blood $(3.5 \mathrm{~mL})$ was carried out in a ROTINA 380R centrifuge (Hettich Zentrifugen, Tuttlingen, Germany) with the tubes positioned at $45^{\circ}$ relative to the rotor. In the first step, the whole blood was centrifuged at $100 \times g$ and $800 \times g$ for $10 \mathrm{~min}$ at $25^{\circ} \mathrm{C}$, followed by the collection of the upper and middle layers using an automatic pipette to obtain the L-PRP 100 $(1.6 \mathrm{~mL})$ and L-PRP $800(2.2 \mathrm{~mL})$. In the second step, L-PRP 100 and L-PRP 800 were centrifuged at $400 \times g$ for $10 \mathrm{~min}$ at $25{ }^{\circ} \mathrm{C}$, and $70 \%$ of the supernatant (platelet-poor plasma, PPP) was discarded, thus obtaining L-PRP ${ }_{100-400}(0.48 \mathrm{~mL})$ and L-PRP $800-400(0.66 \mathrm{~mL})$. L-PRPs and the whole blood were taken to an ABX Micros ES 60 hematologic analyzer (Horiba ABX Diagnostics, Montpellier, France) for the measurement of blood components. The experiment was performed in triplicate $(n=3)$, and three measurements were taken for each sample.

\subsection{Concentration Factor Calculation}

The concentration factor of the platelets and leukocytes (including lymphocytes and granulocytes) was calculated relative to their concentration in the whole blood, according to Equation (1):

$$
\text { Concentration facto }=\frac{[\text { Platelet, Leukocyte, Lymphocyte, Granulocyte }]_{L-P R P}}{[\text { Platelet, Leukocyte, Lymphocyte, Granulocyte }]_{\text {Whole blood }}}
$$

\subsection{Fibrin Nanofiber Formation}

The human whole blood was collected in a tube containing serum clot activator (Greiner Bio-One, Kremsmünster, Austria) and centrifuged at $2000 \times \mathrm{g}$ for $15 \mathrm{~min}$ at $25{ }^{\circ} \mathrm{C}$. After centrifugation, serum-containing thrombin was collected and mixed to $10 \%(w / v) \mathrm{CaCl}_{2}$ (Sigma-Aldrich, St. Louis, MO, USA) in a 9:1 ratio (serum: $\mathrm{Ca}^{2+} v / v$ ). This mixture was added to the L-PRP formulations at a concentration of $20 \%(v / v)$, which were left to polymerize for at least $30 \mathrm{~min}$ to form the fibrin ${ }_{100}$, fibrin $_{800}$, fibrin $100-400$, and fibrin $800-400$ nanofibers.

\subsection{Scanning Electron Microscopy (SEM) Analysis}

After polymerization and the formation of the fibrin nanofibers, the networks were fixed in a $4 \%$ paraformaldehyde and $2.5 \%$ glutaraldehyde solution prepared in phosphate buffer (PBS), pH 7.4, for $2 \mathrm{~h}$ at $4{ }^{\circ} \mathrm{C}$. Dehydration of fixed hydrogels was carried out by dipping them in ethanol of different concentrations $(50 \%, 70 \%, 95 \%$, and $100 \%$ ) at $15 \mathrm{~min}$ intervals. Then, the samples were dried at the critical point in a BAL-TEC critical point dryer (CPD) 030 dryer (Schalksmühle, Germany), coated with gold in a Sputter Coater POLARON, SC7620 (VG Microtech, Uckfield, England), and taken to a LEO 
Electron Microscopy/Oxford (Cambridge, England) for SEM images. Fiber sizes were obtained by measuring at least 100 fibers from three distinct images using the ImageJ software.

\subsection{Kinetics of GFs and Cytokines Release}

Fibrin nanofibers were prepared in a 48-well plate and incubated with low-glucose Dulbecco's modified Eagle's medium (DMEM) for $72 \mathrm{~h}$ in a humidified incubator at $37{ }^{\circ} \mathrm{C}$ and $5 \% \mathrm{CO}_{2}$. At pre-determined times, the medium was collected and stored at $-80{ }^{\circ} \mathrm{C}$. Then, the cumulative concentration of the GFs (TGF- $\beta 1$ and PDGF-BB) and cytokines (IL- 8 and TNF- $\alpha$, ) was quantified using a Bio-plex Pro Kit in a Bio-plex 200 (Bio-Rad, Hercules, CA, USA). The experiment was performed in triplicate $(n=3)$ for each group.

\subsection{Isolation and Culture of h-AdMSCs in Fibrin Nanofibers}

The h-AdMSCs were isolated from the human subcutaneous adipose tissue of patients undergoing lipo-aspiration at the University Hospital, and cultured according to a previous protocol [56]. Cells from passage 5 to 8 were trypsinized and incorporated in each L-PRP at a concentration of $5 \times 10^{4}$ cells $/ \mathrm{mL}$. Then, the L-PRPs were activated with the serum, which was composed of a $\mathrm{Ca}^{2+}$ mixture, and nanofibers containing h-AdMSCs were formed in a 48-well plate and cultured with $750 \mu \mathrm{L}$ of DMEM for 2 weeks in a humidified incubator at $37^{\circ} \mathrm{C}$ and $5 \% \mathrm{CO}_{2}$, with the medium changed every three days. DMEM was not supplemented with fetal bovine serum (FBS) due to the high capacity of L-PRP to provide nutrients for cell survival and growth [57]. The experiment was performed in triplicate $(n=3)$ for each group.

\subsection{Assessment of h-AdMSCs Survival and Growth}

The number of viable cells in the fibrin nanofibers was assessed on the seventh day of culture using a Live/Dead Cell Imaging Kit (Thermo Fisher Scientific, Waltham, MA, USA). Samples were washed with PBS ( $\mathrm{pH} 7.4$ ), incubated with the live/dead reagent for $30 \mathrm{~min}$, and taken to a confocal microscope (Leica Microscope TCS SP5 II, Wetzlar, Germany) for imaging. The numbers of living and dead cells were counted from three different images using ImageJ software. To assess the h-AdMSC proliferation rate, at $3,7,10$, and 14 days, fibrin nanofibers containing the cells were incubated with 1 $\mathrm{mg} / \mathrm{mL}$ dimethyl-thia-zol-2-yl]-2,5-diphenyltetrazolium bromide (MTT) (Sigma-Aldrich, St Louis, MO, USA) for $4 \mathrm{~h}$ at $37^{\circ} \mathrm{C}$. Then, MTT was replaced with dimethyl sulfoxide (DMSO), the samples were left in a shaker for $30 \mathrm{~min}$, and the absorbance was measured at $595 \mathrm{~nm}$. In order to calculate the maximum specific rate of cell growth $\left(\mu_{\max }\right)$ and cell doubling time $\left(t_{d}\right)$, a polynomial curve was adjusted to the experimental absorbance data obtained on days 3,7, and 10. A second-degree polynomial curve adjusted the experimental data with a correlation coefficient of 1 . From the polynomial equations, we determined values at small intervals within the studied range and delineated the cell exponential phase. The $\mu_{\max }$ was calculated using Equation (2), considering the Monod-type kinetic for MSCs [58], and the $t_{d}$ was calculated using Equation (3). The experiment was performed in triplicate $(n=3)$ for each group.

$$
\begin{gathered}
\ln \left[(a b s)_{t} /(a b s)_{0}\right]=\mu_{\max . \mathrm{t}} \\
t_{d}=\frac{\ln 2}{\mu_{\max }}
\end{gathered}
$$

\subsection{Statistical Analysis}

The results are presented here as the mean \pm standard deviation. One-way analysis of variance (ANOVA) with Tukey's test was used with a 95\% confidence level $(p<0.05)$. 
Supplementary Materials: The following are available online at http://www.mdpi.com/1420-3049/24/15/2729/s1, Figure S1: Concentration of (A) platelets, (B) leukocytes, (C) lymphocytes and (D) granulocytes in L-PRPs of the donors 2 and 3, as measured in a hematologic analyzer. The calculated ratios of (E) platelet/leukocyte from $(\mathbf{A}, \mathbf{B})$ and (F) lymphocyte/granulocyte from (C,D). Note: ${ }^{*} p<0.05$. Figure S2: Kinetics of the GFs (A) TGF- $\beta 1$, (B) PDGF-BB, and the inflammatory cytokines (C) IL-8 and (D) TNF- $\alpha$ released for $72 \mathrm{~h}$ from fibrin nanofibers prepared using the L-PRP of two distinct donors (donor 2 and donor 3). The profiles show the strong influence of the centrifugation condition on the soluble factors release and the reproducibility of the tendencies between the donors.

Author Contributions: B.A.G.M. wrote the manuscript, designed and performed the experiments, and analyzed the data. Â.C.M.L. and J.F.S.D.L. provided the basis for the original research concept. M.H.A.S. designed the experiment, supervised the project, and revised the manuscript.

Funding: This work was funded by the São Paulo Research Foundation (FAPESP), grant numbers 2015/23134-8 and 2016/10132-0 and by the National Council for Scientific and Technological Development (CNPq), grant number 308313/2018-6.

Acknowledgments: The authors gratefully acknowledge Prof. Dr. William Dias Belangero for the use of the BAL-TEC CPD 030 dryer and Dr. Adriana da Silva Santos Duarte for her helpful assistance with the h-AdMSC isolation and culture.

Conflicts of Interest: The authors declare no conflicts of interest.

\section{References}

1. Cieslik-Bielecka, A.; Skowroński, R.; Jedrusik-Pawłowska, M.; Pierchała, M. The application of L-PRP in AIDS patients with crural chronic ulcers: A pilot study. Adv. Med. Sci. 2018, 63, 140-146. [CrossRef]

2. Filardo, G.; Kon, E.; Teresa, M.; Ruiz, P.; Cenacchi, A.; Maria, P.; Maurilio, F. Platelet-rich plasma intra-articular injections for cartilage degeneration and osteoarthritis: single- versus double-spinning approach. Knee Surg Sport. Traumatol Arthrosc 2012, 20, 2082-2091. [CrossRef]

3. Lana, J.F.S.D.; Weglein, A.; Sampson, S.; Vicente, E.F.; Huber, S.C.; Souza, C.V.; Ambach, M.A.; Vincent, H.; Urban-Paffaro, A.; Onodera, C.M.K.; et al. Randomized controlled trial comparing hyaluronic acid, platelet-rich plasma and the combination of both in the treatment of mild and moderate osteoarthritis of the knee. J. Stem Cells Regen. Med. 2016, 12, 1-10.

4. Riboh, J.C.; Saltzman, B.M.; Yanke, A.B.; Fortier, L.; Cole, B.J. Effect of Leukocyte Concentration on the Efficacy of Platelet-Rich Plasma in the Treatment of Knee Osteoarthritis. Am. J. Sports Med. 2015, 44, 792-800. [CrossRef]

5. Zotti, F.; Albanese, M.; Rodella, L.F.; Nocini, P.F. Platelet-rich plasma in treatment of temporomandibular joint dysfunctions: Narrative review. Int. J. Mol. Sci. 2019, 20, 277. [CrossRef]

6. Pravin, A.J.S.; Sridhar, V.; Srinivasan, B.N. Autologous Platelet Rich Plasma (Prp) Versus Leucocyte-Platelet Rich Fibrin (L-Prf) in Chronic Non-Healing Leg Ulcers-a Randomised, Open Labelled, Comparative Study. J. Evol. Med. Dent. Sci. 2016, 5, 7460-7462. [CrossRef]

7. Andia, I.; Maffulli, N. Platelet-rich plasma for managing pain and. Nat. Rev. Rheumatol. 2013, 9, 721-730. [CrossRef]

8. Anitua, E.; Sanchez, M.; De la Fuente, M.; Zalduendo, M.M.; Orive, G. Plasma rich in growth factors (PRGF-Endoret) stimulates tendon and synovial fibroblasts migration and improves the biological properties of hyaluronic acid. Knee Surgery, Sport. Traumatol. Arthrosc. 2012, 20, 1657-1665. [CrossRef]

9. Bielecki, T.; Ehrenfest, D.M.D.; Everts, P.A.; Wiczkowski, A. The Role of Leukocytes from L-PRP/L-PRF in Wound Healing and Immune Defense: New Perspectives. Curr. Pharm. Biotechnol. 2012, 13, 1153-1162. [CrossRef]

10. Karin, M.; Clevers, H. Reparative inflammation takes charge of tissue regeneration. Nature 2016, 529, $307-315$. [CrossRef]

11. Rohman, G.; Langueh, C.; Ramtani, S.; Lataillade, J.; Lutomski, D.; Senni, K.; Changotade, S. The Use of Platelet-Rich Plasma to Promote Cell Recruitment into Low-Molecular-Weight. Polymers. 2019, 1-22.

12. Jubert, N.J.; Rodríguez, L.; Reverté-Vinaixa, M.M.; Navarro, A. Platelet-Rich Plasma Injections for Advanced Knee Osteoarthritis. A Prospective, Randomized, Double-Blinded Clinical Trial. Orthop. J. Sport. Med. 2017, 5, 1-11. [CrossRef]

13. Middleton, K.K.; Barro, V.; Muller, B.; Terada, S.; Fu, F.H. Evaluation of the Effects of Platelet-Rich Plasma (PRP) Therapy Involved in the Healing of Sports-Related Soft Tissue Injuries. Iowa Orthop. J. 2012, 32, 150-163. 
14. Zhou, Y.; Zhang, J.; Wu, H.; Hogan, M.V.; Wang, J.H. The differential effects of leukocyte-containing and pure platelet-rich plasma (PRP) on tendon stem/progenitor cells-implications of PRP application for the clinical treatment of tendon injuries. Stem Cell Res. Ther. 2015, 1-13. [CrossRef]

15. Delong, J.M.; Russell, R.P.; Mazzocca, A.D. Platelet-rich plasma: The PAW classification system. Arthrosc. J. Arthrosc. Relat. Surg. 2012, 28, 998-1009. [CrossRef]

16. Ehrenfest, D.M.D.; Andia, I.; Zumstein, M.A.; Zhang, C.-Q.; Pinto, N.R.; Bielecki, T. Classification of platelet concentrates (Platelet-Rich Plasma-PRP, Platelet-Rich Fibrin-PRF) for topical and infiltrative use in orthopedic and sports medicine: current consensus, clinical implications and perspectives. Muscles. Ligaments Tendons J. 2014, 4, 3-9. [CrossRef]

17. Lana, J.F.S.D.; Purita, J.; Paulus, C.; Huber, S.C.; Rodrigues, B.L.; Rodrigues, A.A.; Santana, M.H.; Madureira, J.L.; Malheiros Luzo, Â.C.; Belangero, W.D.; et al. Contributions for classification of platelet rich plasma - Proposal of a new classification: MARSPILL. Regen. Med. 2017, 12, 565-574. [CrossRef]

18. Magalon, J.; Chateau, A.L.; Bertrand, B.; Louis, M.L.; Silvestre, A.; Giraudo, L.; Veran, J.; Sabatier, F. DEPA classification: a proposal for standardising PRP use and a retrospective application of available devices. BMJ Open Sport Exerc. Med. 2016, 2, e000060. [CrossRef]

19. De Melo, B.A.G.; Shimojo, A.A.M.; Perez, A.G.M.; Lana, J.F.S.D.; Santana, M.H.A. Distribution, recovery and concentration of platelets and leukocytes in L-PRP prepared by centrifugation. Colloid Surf. B Biointerfaces 2018, 161, 288-295. [CrossRef]

20. Perez, A.G.M.; Lana, J.F.S.D.; Rodrigues, A.A.; Luzo, A.C.M.; Belangero, W.D.; Santana, M.H.A. Relevant aspects of centrifugation step in the preparation of platelet-rich plasma. ISRN Hematol. 2014, 2014, 176060. [CrossRef]

21. Söderström, A.C.; Nybo, M.; Nielsen, C.; Vinholt, P.J. The effect of centrifugation speed and time on pre-analytical platelet activation. Clin. Chem. Lab. Med. 2016, 54, 1913-1920. [CrossRef] [PubMed]

22. Eyrich, D.; Brandl, F.; Appel, B.; Wiese, H.; Maier, G.; Wenzel, M.; Staudenmaier, R.; Goepferich, A.; Blunk, T. Long-term stable fibrin gels for cartilage engineering. Biomaterials 2007, 28, 55-65. [CrossRef] [PubMed]

23. Wittmann, K.; Dietl, S.; Ludwig, N.; Berberich, O.; Hoefner, C.; Storck, K.; Blunk, K.; Bauer-Kreisel, P. Engineering vascularized adipose tissue using the stromal-vascular fraction and fibrin hydrogels. Tissue Eng. Part A. 2015, 21, 1343-1353. [CrossRef] [PubMed]

24. Snyder, T.N.; Madhavan, K.; Intrator, M.; Dregalla, R.C.; Park, D.; Lawrence, R.; Felson, D.; Helmick, C.; Arnold, L.; Choi, H.; et al. A fibrin/hyaluronic acid hydrogel for the delivery of mesenchymal stem cells and potential for articular cartilage repair. J. Biol. Eng. 2014, 8, 10. [CrossRef] [PubMed]

25. Anitua, E.; Zalduendo, M.; Troya, M.; Padilla, S.; Orive, G. Leukocyte inclusion within a platelet rich plasma-derived fibrin scaffold stimulates a more pro-inflammatory environment and alters fibrin properties. PLoS ONE 2015, 10, 1-19. [CrossRef] [PubMed]

26. Shimojo, A.A.M.; Perez, M.M.G.; Galdames, S.E.M.; Brissac, I.C.S.; Santana, M.H.A. Performance of PRP Associated with Porous Chitosan as a Composite Scaffold for Regenerative Medicine. Sci. World J. 2015, 2015. [CrossRef]

27. Xie, X.; Wang, Y.; Zhao, C.; Guo, S.; Liu, S.; Jia, W.; Tuan, R.S.; Zhang, C. Comparative evaluation of MSCs from bone marrow and adipose tissue seeded in PRP-derived scaffold for cartilage regeneration. Biomaterials 2012, 33, 7008-7018. [CrossRef]

28. Ehrenfest, D.M.D.; Bielecki, T.; Jimbo, R.; Barbe, G.; Del Corso, M.; Inchingolo, F.; Sammartino, G. Do the Fibrin Architecture and Leukocyte Content Influence the Growth Factor Release of Platelet Concentrates? An Evidence-based Answer Comparing a Pure Platelet-Rich Plasma (P-PRP) Gel and a Leukocyte- and Platelet-Rich Fibrin (L-PRF). Curr. Pharm. Biotechnol. 2012, 13, 1145-1152. [CrossRef]

29. Wasterlain, A.S.; Braun, H.J.; Dragoo, J.L. Contents and Formulations of Platelet-Rich Plasma. Oper. Tech. Orthop. 2012, 22, 33-42. [CrossRef]

30. Perez, A.G.M.; Rodrigues, A.A.; Luzo, A.C.M.; Lana, J.F.S.D.; Belangero, W.D.; Santana, M.H.A. Fibrin network architectures in pure platelet-rich plasma as characterized by fiber radius and correlated with clotting time. J. Mater. Sci. Mater. Med. 2014, 25, 1967-1977. [CrossRef]

31. Crane, D.; Everts, P.A.M. Platelet Rich Plasma (PRP) Matrix Grafts. Pract. PAIN Manag. 2008, 1-10.

32. Caplan, A.I.; Correa, D. PDGF in Bone Formation and Regeneration: New Insights into a Novel Mechanism Involving MSCs. J. Orthop. Res. 2011, 1795-1803. [CrossRef] [PubMed] 
33. Xu, X.; Zheng, L.; Yuan, Q.; Zhen, G.; Crane, J.L.; Zhou, X.; Cao, X. Transforming growth factor- $\beta$ in stem cells and tissue homeostasis. Bone Res. 2018, 6. [CrossRef] [PubMed]

34. Chaly, Y.V.; Selvan, R.S.; Fegeding, K.V.; Kolesnikova, T.S.; Voitenok, N.N. Expression of Il-8 Gene in Human Monocytes and Lymphocytes: Differential Regulation by tnf and Il-1. Cytokine 2000, 12, 636-643. [CrossRef] [PubMed]

35. Kapoor, M.; Martel-Pelletier, J.; Lajeunesse, D.; Pelletier, J.P.; Fahmi, H. Role of proinflammatory cytokines in the pathophysiology of osteoarthritis. Nat. Rev. Rheumatol. 2011, 7, 33-42. [CrossRef] [PubMed]

36. Brat, D.J.; Bellail, A.C.; Van Meir, E.G. The role of interleukin- 8 and its receptors in gliomagenesis and tumoral angiogenesis. Neuro. Oncol. 2005, 7, 122-133. [CrossRef] [PubMed]

37. Roman-Blas, J.A.; Stokes, D.G.; Jimenez, S.A. Modulation of TGF- $\beta$ signaling by proinflammatory cytokines in articular chondrocytes. Osteoarthr. Cartil. 2007, 15, 1367-1377. [CrossRef]

38. Wilgus, T.A.; Roy, S.; McDaniel, J.C. Neutrophils and Wound Repair: Positive Actions and Negative Reactions. Adv. Wound Care 2013, 2, 379-388. [CrossRef]

39. Parrish, W.R.; Roides, B.; Hwang, J.; Mafilios, M.; Story, B.; Bhattacharyya, S. Normal platelet function in platelet concentrates requires non-platelet cells: a comparative in vitro evaluation of leucocyte-rich (type 1a) and leucocyte-poor (type 3b) platelet concentrates. BMJ Open Sport Exerc. Med. 2016, 2, e000071. [CrossRef]

40. Sundman, E.A.; Cole, B.J.; Fortier, L.A. Growth Factor and Catabolic Cytokine Concentrations Are Influenced by the Cellular Composition of Platelet-Rich Plasma. Am. J. Sports Med. 2011, 39, 2135-2140. [CrossRef]

41. Barnett Jr, M.D.; Pomeroy, G.C. Use of Platelet-Rich Plasma and Bone Marrow-Derived Mesenchymal Stem Cells in Foot and Ankle Surgery. Tech. Foot Ankle Surg. 2007, 6, 89-94. [CrossRef]

42. Holmes, H.L.; Wilson, B.; Goerger, J.P.; Silverberg, J.L.; Cohen, I.; Zipfel, W.R.; Fortier, L.A. Facilitated recruitment of mesenchymal stromal cells by bone marrow concentrate and platelet rich plasma. PLoS ONE 2018, 13, 1-12. [CrossRef] [PubMed]

43. Jia, J.; Wang, S.; Ma, L.; Yu, J.; Guo, Y.; Wang, C. The Differential Effects of Leukocyte-Containing and Pure Platelet-Rich Plasma on Nucleus Pulposus-Derived Mesenchymal Stem Cells: Implications for the Clinical Treatment of Intervertebral Disc Degeneration. Stem Cells Int. 2018, 2018. [CrossRef] [PubMed]

44. Xu, Z.; Yin, W.; Zhang, Y.; Qi, X.; Chen, Y.; Xie, X.; Zhang, C. Comparative evaluation of leukocyte- and platelet-rich plasma and pure platelet-rich plasma for cartilage regeneration. Sci. Rep. 2017, 7, 43301. [CrossRef] [PubMed]

45. Perez, A.G.M.; Lichy, R.; Lana, J.F.S.D.; Rodrigues, A.A.; Luzo, A.C.M.; Belangero, W.D.; Santana, M.H.A. Prediction and modulation of platelet recovery by discontinuous centrifugation of whole blood for the preparation of pure platelet-rich plasma. Biores. Open Access 2013, 2, 307-314. [CrossRef]

46. Anitua, E.; Andia, I.; Sanchez, M.; Azofra, J.; del Mar Zalduendo, M.; Nurden, P.; Nurden, A.T. Autologous preparations rich in growth factors promote proliferation and induce VEGF and HGF production by human tendon cells in culture. J. Orthop. Res. 2005, 23, 281-286. [CrossRef]

47. Anitua, E.; Sánchez, M.; Nurden, A.T.; Zalduendo, M.M.; Dela Fuente, M.; Azofra, J.; Andía, I. Platelet-released growth factors enhance the secretion of hyaluronic acid and induce hepatocyte growth factor production by synovial fibroblasts from arthritic patients. Rheumatology 2007, 46, 1769-1772. [CrossRef]

48. Choukroun, J.; Ghanaati, S. Reduction of relative centrifugation force within injectable platelet-rich-fibrin (PRF) concentrates advances patients' own inflammatory cells, platelets and growth factors: the first introduction to the low speed centrifugation concept. Eur. J. Trauma Emerg. Surg. 2018, 44, 87-95. [CrossRef]

49. Ehrenfest, D.M.D.; Pinto, N.R.; Pereda, A.; Jiménez, P.; Del Corso, M.; Kang, B.; Nally, M.; Lanata, N.; Quirynen, M.; Dohan, D.M.; et al. The impact of the centrifuge characteristics and centrifugation protocols on the cells, growth factors, and fibrin architecture of a leukocyte- and platelet-rich fibrin (L-PRF) clot and membrane. Platelets 2018, 29, 171-184. [CrossRef]

50. Dohle, E.; El Bagdadi, K.; Sader, R.; Choukroun, J.; Kirkpatrick, C.J.; Ghanaati, S. Platelet-rich fibrin-based matrices to improve angiogenesis in an in vitro co-culture model for bone tissue engineering. J. Tissue Eng. Regen. Med. 2017, 1-13. [CrossRef]

51. Hutton, D.L.; Moore, E.M.; Gimble, J.M.; Grayson, W.L. Platelet-Derived Growth Factor and Spatiotemporal Cues Induce Development of Vascularized Bone Tissue by Adipose-Derived Stem Cells. TISSUE Eng. Part. A 2013, 19, 2076-2086. [CrossRef] [PubMed] 
52. Smyth, M.J.; Zachariae, C.C.; Norihisa, Y.; Ortaldo, J.R.; Hishinuma, A.; Matsushimat, A.N.D.K. IL-8 gene expression and production in human peripheral blood lymphocyte subsets. J. Immunol. 1991, 146, 3815-3823. [PubMed]

53. Dubravec, D.B.; Spriggst, D.R.; Mannick, J.A.; Rodrick, M.L. Circulating human peripheral blood granulocytes synthesize and secrete tumor necrosis factor a. Proc. Natl. Acad. Sci. USA 1990, 87, 6758-6761. [CrossRef] [PubMed]

54. Taub, D.D.; Anver, M.; Oppenheim, J.J.; Longo, D.L.; Murphy, W.J. T lymphocyte recruitment by interleukin-8 (IL-8). IL-8-induced degranulation of neutrophils releases potent chemoattractants for human Tymphocytes both in vitro and in vivo. J. Clin. Invest. 1996, 97, 1931-1941. [CrossRef] [PubMed]

55. Ng, F.; Boucher, S.; Koh, S.; Sastry, K.S.R.; Chase, L.; Lakshmipathy, U.; Choong, C.; Yang, Z.; Vemuri, M.C.; Rao, M.S.; et al. PDGF, tgf-beta and FGF signaling is important for differentiation and growth of mesenchymal stem cells (MSC): Transcriptional profiling can identify markers and signaling pathways important in differentiation of MSCs into adipogenic, chondrogenic, and ost. Blood 2008, 112, 295-307. [CrossRef] [PubMed]

56. Manzini, B.M.; da Silva Santos Duarte, A.; Sankaramanivel, S.; Ramos, A.L.; Latuf-Filho, P.; Escanhoela, C.; Kharmandayan, P.; Olalla Saad, S.T.; Boin, I.; Malheiros Luzo, Â.C. Useful properties of undifferentiated mesenchymal stromal cells and adipose tissue as the source in liver-regenerative therapy studied in an animal model of severe acute fulminant hepatitis. Cytotherapy 2015, 17, 1052-1065. [CrossRef] [PubMed]

57. Murphy, M.B.; Blashki, D.; Buchanan, R.M.; Yazdi, I.K.; Ferrari, M.; Simmons, P.J.; Tasciotti, E. Adult and umbilical cord blood-derived platelet-rich plasma for mesenchymal stem cell proliferation, chemotaxis, and cryo-preservation. Biomaterials 2012, 33, 5308-5316. [CrossRef]

58. Higuera, G.; Schop, D.; Janssen, F.; van Dijkhuizen-Radersma, R.; van Boxtel, T.; van Blitterswijk, C.A. Quantifying In Vitro Growth and Metabolism Kinetics of Human Mesenchymal Stem Cells Using a Mathematical Model. Tissue Eng. Part. A 2009, 15, 2653-2663. [CrossRef]

Sample Availability: Samples of the compounds are not available from the authors.

(C) 2019 by the authors. Licensee MDPI, Basel, Switzerland. This article is an open access article distributed under the terms and conditions of the Creative Commons Attribution (CC BY) license (http://creativecommons.org/licenses/by/4.0/). 